\title{
Condutividade hidráulica e atributos físicos de um Latossolo Vermelho sob sistemas de manejo no Cerrado Goiano
}

\section{Hydraulic conductivity and physical attributes of an Oxisol under management systems in the Goiás Cerrado}

Carlos Augusto Oliveira de Andrade

Universidade Federal de Goiás

E-mail: carlosandradeuft@hotmail.com

OrclD: https://orcid.org/0000-0001-5836-2854

Gustavo Cassiano da Silva

Universidade Federal de Goiás

E-mail: gustavo.cassred@hotmail.com

OrcID: https://orcid.org/0000-0001-9913-8779

Marcos Costa Corrêa

Universidade Federal de Goiás

E-mail: venezaengenharia@gmail.com

OrcID: https://orcid.org/0000-0001-9058-8917

Leonardo Santos Collier

Universidade Federal de Goiás

E-mail: collierufg@gmail.com

OrcID: https://orcid.org/0000-0002-6911-8037

Vladia Correchel

Universidade Federal de Goiás

E-mail: vladiaea@ufg.br

OrcID: https://orcid.org/0000-0001-7109-2588

Resumo: Os solos quando submetidos a sistemas de cultivos, tendem a um novo estado de equilíbrio, apresentando distintas manifestações de seus atributos. $O$ objetivo deste trabalho foi avaliar as modificações provocadas por sistemas de uso do solo sobre a condutividade hidráulica e atributos físicos de um Latossolo Vermelho localizado em área de Cerrado. Foram analisados três sistemas: Cerrado Nativo (CN) - sem intervenções antrópicas, Sistema Agroflorestal (SAF) - composto por árvores de Dipteryx alata V. consorciadas com culturas anuais e perenes em sistema rotacionado, implantado no ano de 2006 e Pastagem - formada por Brachiaria brizantha com sistema de cultivo extensivo desde 1997. Foi utilizado o delineamento inteiramente casualizado, com 5 repetições. Foram avaliadas a condutividade hidráulica do solo saturado (Ks), densidade (Ds), porosidade total (Pt), resistência a penetração (Rp) e a umidade do solo, em três profundidades $0-0,10 ; 0,10-0,20$ e 0,20-0,40 m. Os dados foram submetidos à análise de variância e a comparação de médias foi feita pelo teste de Tukey $(p<0,05)$. Sete anos de cultivo no SAF propiciaram manutenção dos níveis de Ks e Rp nos primeiros $0,10 \mathrm{~m}$ de profundidade, o que indica qualidade física do solo pensando no desenvolvimento das espécies agrícolas. Pelo pouco tempo de manejo, intensidade de cultivo e baixa diversidade de espécies arbóreas presentes no SAF, ainda não foi possível verificar melhorias dos atributos físicos nas camadas subsuperficiais, sendo comparado ao observado na área de pastagem. Os valores dos atributos físicos mensurados para os sistemas, apesar de inferiores ao $\mathrm{CN}$, não permitem caracterizar um quadro de degradação intensa dos solos. 
Palavras-chave: agrofloresta, cerrado nativo, forragens, qualidade física do solo, sistema conservacionista

\begin{abstract}
Soils submitted to cropping systems tend to a new state of equilibrium, reflected in different manifestations of their attributes. The objective of this work was to evaluate the modifications caused by soil use systems on the saturated hydraulic conductivity and physical attributes of an Oxisol of Cerrado. Three systems of soil use were analyzed: Native Cerrado (CN) - without anthropic interventions, Agroforestry System (SAF) - composed of Dipteryx alata V. trees, intercropped with annual and perennial crops in a rotational system, implanted in 2006 and Pasture - formed by Brachiaria brizantha with extensive cultivation system since 1997. A completely randomized design with 5 replications was used. Were evaluated the saturated hydraulic conductivity (Ks), global density (Ds), total porosity (Pt), penetration resistance (Rp) and soil moisture in three layers $0-0,10 ; 0,10-0,20$ e $0,20-0,40 \mathrm{~m}$. The data were subjected to analysis of variance and the comparison of means was performed by the Tukey test $(p<0,05)$. Seven years of cultivation in the SAF provided maintenance of the Ks and Rp levels in the first $0,10 \mathrm{~m}$ of depth, which indicates physical soil quality considering the development of agricultural species. Due to the short time of management, intensity of cultivation and low diversity of tree species present in the SAF, it has not yet been possible to verify improvements in physical attributes in the subsurface layers, being compared to that observed in the pasture area. The values of the physical attributes measured for the systems, although lower than the CN, do not allow to characterize a picture of intense soil degradation.
\end{abstract}

Keywords: agroforestry, brazilian cerrado, forages, physical soil quality, conservationist system

Data de recebimento: 01/03/2019

Data de aprovação: 08/06/2020

DOI: https://doi.org/10.30612/agrarian.v13i49.9519

\title{
Introdução
}

A região Centro-Oeste, onde o Cerrado é o bioma predominante, é caracterizada por grandes extensões de solos profundos, bem drenados, com características físicas e topográficas favoráveis a intensa mecanização agrícola (Manzatto et al., 2002). A classe de solos com maior ocorrência no Cerrado são os Latossolos, que apesar de apresentarem condições físicas favoráveis para a produção agrícola (Ker, 1997; Freitas et al., 2014), possuem desvantagens para as características químicas, apresentando, em geral, baixa fertilidade natural, acidez acentuada e reduzido teor de matéria orgânica (Cunha et al., 2008).

Os solos do Cerrado, quando manejados de maneira inadequada, podem limitar a capacidade produtiva e favorecer o processo de degradação. O modelo convencional de exploração agropecuária com uso intensivo de arado de disco e grade aradora para o preparo do solo, além de cultivos monoculturais e pastagens extensivas, têm contribuído nesse processo (Jorge et al., 2012), resultando em compactação do solo, erosão, assoreamento de rios e perda de biodiversidade, com reflexos sobre todo o ecossistema (Cunha et al., 2008).

O conhecimento dos danos provocados pelos diferentes tipos de manejo é essencial para determinação da escolha dos sistemas produtivos favoráveis à conservação da capacidade produtiva e melhoria da qualidade física do solo. A dificuldade de associar produção agropecuária com conservação do solo incentivou pesquisadores a desenvolverem várias técnicas conservacionistas de manejos a fim de evitar a degradação física do solo e aumentar a sustentabilidade dos cultivos (Oliveira et al., 2015).

Dentre as técnicas conservacionistas os Sistemas Agroflorestais (SAFs) se destacam, por ser uma alternativa associada de recuperação de áreas degradadas com produção agropecuária. Esses sistemas são caracterizados pela combinação integrada de árvores, arbustos, cultivos agrícolas com presença ou não de animais em uma mesma área, de maneira simultânea ou sequencial (Pezarico et al., 2013), o que favorece maior interação entre as espécies, aumenta a biodiversidade vegetal e propicia o uso mais eficiente dos nutrientes e da água do solo (Arruda, 2017). 
A utilização de SAF tem sido, nas últimas décadas, bastante difundida como alternativa para recuperação de áreas degradadas e, sua adoção, tem colaborado com melhorias químicas e físicas do solo, com consequente incremento da matéria orgânica e decréscimo dos processos erosivos, incremento na qualidade da água e conservação da biodiversidade (Nair, 2008).

As relações entre os sistemas de manejo e a qualidade do solo podem ser avaliadas pelas modificações de seus atributos, dentre eles os físicos, os quais têm sido recomendados por diversos autores (Carneiro et al., 2009; Torres et al., 2015). Esses atributos exercem influência em outros parâmetros do solo, como a taxa de infiltração e a capacidade de retenção de água, o que reflete diretamente na perda de água e solo pelos sistemas de manejo, ocasionando em maior ou menor grau o processo de degradação (Ramos et al., 2014; Beniaich et al., 2020). Nesse contexto, para a identificação e desenvolvimento de sistemas agrícolas sustentáveis, são necessários estudos que contribuam para a compreensão do impacto do uso e manejo do solo na sua qualidade física.

Assim, o objetivo deste trabalho foi avaliar as modificações provocadas por sistemas de uso do solo na condutividade hidráulica do solo saturado e nos atributos físicos de um Latossolo Vermelho sob Cerrado em Goiânia-GO.

\section{Material e Métodos \\ Caracterização da área de estudo}

A pesquisa foi desenvolvida no campus experimental da Escola de Agronomia da Universidade Federal de Goiás, localizada em Goiânia, Goiás, Brasil, às coordenadas 16³6"11'S e 49¹6"47'O, com altitude média local de $730 \mathrm{~m}$. O clima da região, segundo a classificação de Köppen, é do tipo Aw (Megatérmico), com invernos secos e verões chuvosos, apresentando precipitação média anual de $1.485 \mathrm{~mm}$ e temperatura média anual de $25^{\circ} \mathrm{C}$. O solo da área foi classificado como LATOSSOLO VERMELHO Ácrico típico, textura argilo-arenosa, A moderado (Santos et al., 2013).

Foram estudados três sistemas de uso do solo: 1. Cerrado nativo (CN)-Cerrado preservado com 3,32 hectares de extensão, formado por espécies vegetais típicas do bioma e sem intervenções antrópicas; 2 . Sistema agroflorestal (SAF)- implantado no ano de 2006, composto por árvores de Baru (Dipteryx alata V.) plantadas com espaçamento de 6 metros entre linhas e 3 entre plantas, com tamanho total de 0,2 ha, sendo que a partir do ano de 2010, entre os renques dos barueiros, têm sido cultivadas culturas anuais e perenes como feijão guandu (Cajanus cajan), estilosantes (Stylosanthes capitata), capim massai (Panicum maximum), milho (Zea mays) e banana (Musa spp.), de maneira consorciada e rotacionada e, 3. Pastagem - formada por Urochloa brizantha, possuindo 3,36 ha, predominando o sistema extensivo de pastejo desde o ano de 1997.

$\mathrm{Na}$ ocasião da implantação do SAF e da pastagem, o solo foi preparado com uso de grade aradora 16x28" e aplicação de 1,5 t de calcário, incorporados até $0,20 \mathrm{~m}$ de profundidade por meio de uma segunda gradagem, com objetivo de elevar a saturação de bases a $50 \%$. Após a implantação, a área do SAF permaneceu sem revolvimento, adotando o cultivo mínimo para as culturas subsequentes, sendo as adubações realizadas de acordo com as recomendações de Sousa e Lobato (2004). Para a pastagem, a área não recebeu nenhuma outra operação de manejo da fertilidade após sua implantação.

\section{Coleta de amostras e indicadores avaliados}

De outubro a dezembro de 2017 foram coletadas amostras indeformadas de solo em anéis volumétricos com dimensões de $50 \mathrm{~mm}$ de altura $\times 50 \mathrm{~mm}$ de diâmetro, extraídos por meio de um amostrador de Uhland, para a determinação das análises físicas estruturais do solo. As coletas foram realizadas em cinco locais aleatórios para cada sistema de uso do solo, nas profundidades $0-0,10 ; 0,10-0,20$ e $0,20-0,40$ m. Nos mesmos locais e nas mesmas profundidades, foram determinados à condutividade hidráulica do solo e a resistência do solo a penetração.

A densidade do solo (Ds) foi calculada pela relação massa/volume. Para isso, as amostras de solo foram levadas para estufa a $105^{\circ} \mathrm{C}$ por aproximadamente 48 horas, até peso constante (Blake \& Hartge, 1986). 
A porosidade total do solo $(\mathrm{Pt})$ foi estimada pelo método indireto, através da fórmula $\mathrm{Pt}=(\mathrm{Dp}-$ Ds/Dp) de acordo com Vomocil (1965). Para fins deste cálculo foram determinados também os valores de densidade de partículas (Dp) pelo método do balão volumétrico (Klute, 1986).

A umidade atual do solo foi determinada em base gravimétrica pelo método clássico de pesagem, empregando a metodologia proposta por Uhland (1951).

A condutividade hidráulica do solo saturado foi quantificada em campo usando-se o permeâmetro de Guelph. Para isso, foi medida a taxa de infiltração constante em campo e calculada a Ks segundo Reynolds e Elrick (1987), através da equação [1]:

$K s=\frac{Q C}{2 \pi H^{2}+C \pi R^{2}+2 \pi \frac{H}{A}}$

Em que: Ks $\left(\mathrm{mm} \mathrm{h}^{-1}\right)$ é a condutividade hidráulica do solo saturado a campo, $Q\left(\mathrm{~m}^{3} \mathrm{~s}^{-1}\right)$ é a vazão constante infiltrada, $C$ é fator adimensional, estabelecido em função da relação $H / A$, onde $H(m)$ é a carga hidráulica utilizada, $A\left(\mathrm{~cm}^{-1}\right)$ é um parâmetro associado ao tipo de solo e $R(\mathrm{~m})$ é o raio do orifício aberto pelo trado no solo.

A resistência do solo a penetração $(R p)$ foi estimada em campo com o penetrômetro de impacto modelo IAA/Planalsucar, sendo os valores de índice de cone transformados em Rp conforme procedimentos descritos em Stolf et al. (2014).

\section{Delineamento experimental e análise estatística dos dados}

A pesquisa foi conduzida em delineamento inteiramente casualizado, com cinco repetições em cada tratamento (sistema x profundidades). Os resultados da condutividade hidráulica e dos atributos físicos do solo foram submetidos à análise de variância pelo teste $F$ ao nível de $p<0,01$ e $p<0,05$ e, quando apropriado, a comparação das médias se deu por meio do teste Tukey a $p<0,05$, utilizando o programa estatístico Sisvar versão 5.6 (Ferreira, 2011).

\section{Resultados e Discussão}

A condutividade hidráulica do solo saturado (Ks) apresentou resposta distinta entre os sistemas de uso do solo e as profundidades estudadas (Tabela 1). Os maiores valores de Ks foram encontrados nas

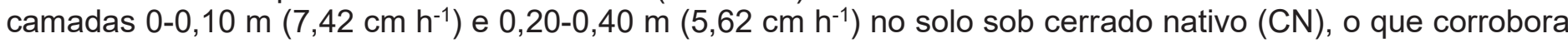
com os resultados obtidos por Rossetti e Centurion (2013) que, estudando as modificações provocadas por diferentes sistemas de manejo nos atributos físico-hídricos em um Latossolo Vermelho, verificaram maiores valores de Ks próximos à superfície do solo para a área nativa, quando comparada com sistema de plantio direto com 10 anos de uso.

$\mathrm{Na}$ camada 0-0,10 m o sistema agroflorestal (SAF) indicou valor de Ks mais próximo ao do CN, considerado como referencial e maior que o da pastagem. Por outro lado, em todos os tratamentos, foi constatada tendência de diminuição da Ks com o aumento da profundidade, excetuando a pastagem que mostrou o menor valor médio $\left(1,71 \mathrm{~cm} \mathrm{~h}^{-1}\right)$ na camada 0-0,10 m (Tabela 1).

Esses resultados revelam que apesar do pouco tempo de implantação do SAF (7 anos), as práticas adotadas como revolvimento mínimo do solo, a diversidade de culturas cultivadas e a diversificação de sistemas radiculares, provavelmente incrementaram a Ks nos primeiros centímetros de solo. De maneira contrária, a diminuição da Ks na pastagem pode estar associada com a maior compactação em superfície, provocada pelo pisoteio animal durante o pastejo, ocasionando menor fluxo saturado da água (Cardoso et al., 2011). Segundo Torres et al. (2011) e Hickmann et al. (2012), a formação de bioporos por meio da presença de organismos edáficos e os espaços deixados a partir da morte do sistema radicular das culturas antecessoras, possibilitam a produção de poros contínuos que facilitam a condução da água no perfil do solo.

Agrarian, Dourados, v. 13, n. 49, p. 385-392, 2020. 
Tabela 1. Condutividade hidráulica do solo saturado $\left(\mathrm{cm} \mathrm{h}^{-1}\right)$, densidade do solo $\left(\mathrm{Mg} \mathrm{m}^{-3}\right)$, porosidade total $\left(\mathrm{m}^{3} \mathrm{~m}^{-3}\right)$, resistência a penetração $(\mathrm{MPa})$ e umidade do solo $\left(\mathrm{kg} \mathrm{kg}^{-1}\right)$ em função dos diferentes sistemas de uso do solo e profundidades estudadas

\begin{tabular}{|c|c|c|c|}
\hline \multirow{3}{*}{ Tratamentos } & \multicolumn{3}{|c|}{ Profundidade $(\mathrm{m})$} \\
\hline & $0-0,10$ & $0,10-0,20$ & $0,20-0,40$ \\
\hline & \multicolumn{3}{|c|}{ Condutividade hidráulica do solo saturado $\left(\mathrm{cm} \mathrm{h}^{-1}\right)$} \\
\hline Cerrado nativo & $7,42 \mathrm{Aa}$ & $5,08 \mathrm{Ab}$ & $5,62 \mathrm{Ab}$ \\
\hline Sistema agroflorestal & $5,61 \mathrm{Ba}$ & $2,60 \mathrm{Ab}$ & $2,11 \mathrm{Bb}$ \\
\hline \multirow{2}{*}{ Pastagem } & $1,71 \mathrm{Cb}$ & $3,20 \mathrm{Aa}$ & $2,30 \mathrm{Bab}$ \\
\hline & \multicolumn{3}{|c|}{ Densidade do solo $\left(\mathrm{Mg} \mathrm{m}^{-3}\right)$} \\
\hline Cerrado nativo & $1,13 \mathrm{Ba}$ & $1,15 \mathrm{Ca}$ & $1,19 \mathrm{Ba}$ \\
\hline Sistema agroflorestal & $1,26 \mathrm{Ac}$ & $1,43 \mathrm{Aa}$ & $1,35 \mathrm{Ab}$ \\
\hline \multirow{2}{*}{ Pastagem } & $1,33 \mathrm{Aa}$ & $1,36 \mathrm{Ba}$ & $1,20 \mathrm{ABb}$ \\
\hline & \multicolumn{3}{|c|}{ Porosidade total do solo $\left(\mathrm{m}^{3} \mathrm{~m}^{-3}\right)$} \\
\hline Cerrado nativo & $0,576 \mathrm{Aa}$ & $0,575 \mathrm{Aa}$ & $0,560 \mathrm{Aa}$ \\
\hline Sistema agroflorestal & $0,569 \mathrm{ABa}$ & $0,518 \mathrm{Bb}$ & $0,528 \mathrm{Ab}$ \\
\hline \multirow[t]{2}{*}{ Pastagem } & $0,534 \mathrm{Bb}$ & $0,523 \mathrm{Bb}$ & $0,575 \mathrm{Aa}$ \\
\hline & \multicolumn{3}{|c|}{ Resistência a penetração (MPa) } \\
\hline Cerrado nativo & $1,19 \mathrm{Bb}$ & $1,21 \mathrm{Cb}$ & $1,45 \mathrm{Ba}$ \\
\hline Sistema agroflorestal & $1,18 \mathrm{Bc}$ & $2,37 \mathrm{Aa}$ & $2,04 \mathrm{Ab}$ \\
\hline \multirow[t]{2}{*}{ Pastagem } & $2,36 \mathrm{Aa}$ & $2,21 \mathrm{Ba}$ & $2,10 \mathrm{Aa}$ \\
\hline & \multicolumn{3}{|c|}{ Umidade do solo $\left(\mathrm{kg} \mathrm{kg}^{-1}\right)$} \\
\hline Cerrado nativo & $0,252 \mathrm{Aa}$ & $0,263 \mathrm{Aa}$ & $0,265 \mathrm{Aa}$ \\
\hline Sistema agroflorestal & $0,236 \mathrm{Aa}$ & $0,227 \mathrm{Ba}$ & $0,236 \mathrm{Ba}$ \\
\hline Pastagem & $0,245 \mathrm{Aa}$ & $0,233 \mathrm{Ba}$ & $0,245 \mathrm{ABa}$ \\
\hline
\end{tabular}

Médias seguidas de mesma letra, maiúsculas nas colunas e minúsculas nas linhas, não diferem entre si pelo teste de Tukey $(p<0,05)$

Em relação à densidade do solo (Ds), houve diferenças entre os sistemas de uso do solo, para as três profundidades avaliadas (Tabela 1). O CN apresentou os menores valores de Ds dentre as áreas para todas as profundidades, diferindo do SAF e da pastagem nas profundidades $0-0,10$ e $0,10-0,20 \mathrm{~m}$ e apenas do primeiro na profundidade $0,20-0,40 \mathrm{~m}$. Por outro lado, comparando o SAF com a pastagem, observou-se diferença apenas na camada $0,10-0,20 \mathrm{~m}$, onde foi constatado maior valor de Ds para o SAF $\left(1,43 \mathrm{Mg} \mathrm{m}^{-3}\right)$.

$\mathrm{Na}$ avaliação da porosidade total do solo $(\mathrm{Pt})$ foram observados decréscimos dos valores para o SAF e a pastagem em relação ao $\mathrm{CN}$, nas profundidades $0-0,10$ e 0,10-0,20 m (Tabela 1). No entanto, para os primeiros centímetros do solo (0-0,10 m), o SAF apresentou similaridade de poros com o CN, resposta que não ocorreu na pastagem. Em relação às profundidades avaliadas, o SAF indicou redução da $\mathrm{Pt}$ com o aumento da profundidade, enquanto a pastagem apresentou resposta contrária.

Resultados semelhantes foram encontrados por Aratani et al. (2009) trabalhando com qualidade física de um Latossolo Vermelho acriférrico em áreas conduzidas em sistema plantio direto com diferentes tempos de implantação, sistema de preparo convencional e mata nativa, observaram dentre todos os tratamentos, menores valores de Ds e maiores valores de macroporosidade e porosidade total, para a área da mata, atribuindo esses resultados a preservação dos atributos físicos, ocasionado pela ausência de ações antrópicas no ambiente.

Quanto à resistência a penetração $(\mathrm{Rp})$, foi observado o maior valor para a pastagem na camada 0$0,10 \mathrm{~m}$ (2,36 MPa), apresentando diferença para os demais sistemas, não sendo observado, portanto, variação nos valores de umidade (Tabela 1). Na profundidade 0,10-0,20 m, tanto o SAF, quanto a pastagem, indicaram valores elevados de $\operatorname{Rp}(2,37$ e 2,21 MPa, respectivamente) e decréscimo na umidade do solo em relação ao CN. Essa mesma resposta foi observada na profundidade 0,20-0,40 m, indicando maior grau de compactação na área sob pastagem, desde os primeiros centímetros de solo até a profundidade $0,40 \mathrm{~m}$, resultado que também foi constatado no SAF, porém, apenas na profundidade intermediária $(0,10-0,20 \mathrm{~m})$. Vários autores (Costa et al., 2012; Moreira et al., 2012) consideram valores de Rp acima de 2,0 MPa prejudiciais ao desenvolvimento do sistema radicular das culturas, ocorrendo por consequência a diminuição do fluxo de água e ar no solo. 
Essas variações dos resultados observados nos parâmetros físicos avaliados, para os sistemas de uso do solo e profundidades, podem ser explicadas pelas características de manejo de cada sistema. $\mathrm{Na}$ pastagem o maior valor de Ds e resistência do solo a penetração em superfície, provavelmente foram influenciados pelo manejo extensivo adotado para o sistema, sendo o superpastejo, o pisoteio animal e a falta de mobilização do solo os principais fatores responsáveis pelos incrementos (Torres et al., 2011). Isso compromete a sustentabilidade da produção e promove inúmeros danos no solo e nas plantas como, diminuição da taxa de infiltração, aumento da erosão e impedimento do crescimento radicular em profundidade (Marchão et al., 2007).

No SAF, a maior Ds e resistência à penetração observada na profundidade $0,10-0,20 \mathrm{~m}$, juntamente com menor porosidade, demonstra início de compactação, podendo ser explicada pelo preparo do solo mais recente entre os sistemas avaliados, realizado na sua fase de implantação, onde o uso de máquinas e implementos de preparo favoreceu a formação desta zona compactada (Torres et al., 2011). Esperava-se que a utilização de cultivos consorciados, manutenção de material vegetal na superfície e a rotação de culturas adotados nesse sistema, alterassem os atributos físicos do solo para essa camada. No entanto, devido ao pouco tempo de implantação desse sistema na área e a baixa diversidade de espécies arbóreas cultivadas, os benefícios em profundidade podem ainda não ter se expressado, como constatado na camada superficial.

Para o CN, os menores valores de Ds e resistência à penetração e maiores valores de porosidade e umidade nas profundidades estudadas, podem ser atribuídos à conservação dos atributos físicos do solo, aliados a diversidade de raízes e ao acúmulo de matéria orgânica presentes nesse ambiente natural (Leroy et al., 2008). Pois, em sistemas nativos, inalterados pelas ações antrópicas, as propriedades físicas do solo são preservadas (Hickmann et al., 2012) e os sistemas radiculares mais conservados e em maior quantidade, contribuem para a agregação das partículas do solo, produzindo aumento da porosidade e menores valores de densidade do solo (Ramos et al., 2010). Além disso, o maior conteúdo de material orgânico presente nesse sistema favorece a diminuição das perdas de água por evaporação, conservando a umidade do solo por mais tempo (Solino et al., 2010).

\section{Conclusões}

Sete anos de cultivo agrícola no sistema agroflorestal (SAF) propiciaram manutenção dos níveis de condutividade hidráulica e resistência do solo à penetração nos primeiros $0,10 \mathrm{~m}$ de profundidade, o que indica qualidade física do solo pensando no desenvolvimento das espécies agrícolas.

Pelo pouco tempo de manejo, intensidade de cultivo e baixa diversidade de espécies arbóreas presentes no SAF, ainda não foi possível verificar uma melhoria dos atributos físicos do solo nas camadas subsuperficiais, sendo comparado ao observado na área com 20 anos de pastagem.

Os valores dos atributos físicos mensurados, principalmente de resistência a penetração, apesar de inferiores ao cerrado nativo (CN) não permitem caracterizar um quadro de degradação intensa dos solos.

\section{Referências}

ARATANI, R. G.; FREDDI, O. S.; CENTURION, J. F.; ANDRIOLI, I. Qualidade física de um Latossolo Vermelho Acriférrico sob diferentes sistemas de uso e manejo. Revista Brasileira de Ciência do Solo, v. 33, n. 3, p. 677-687, 2009.

ARRUDA, E. M. Atributos químicos do solo e desempenho de culturas em rotação ou consórcio com plantas de cobertura em sistema agroflorestal. 2017. Ano de obtenção: 2017. 88 f. Tese (Doutorado em Agronomia: Solo e Água), Universidade Federal de Goiás, 2017.

BENIAICH, A.; SILVA, M. L. N.; GUIMARÃES, D. V.; BISPO D. F. A.; AVANZI, J. C.; CURI, N.; PIO, R.; DONDEYNE, S. Assessment of soil erosion in olive orchards (Olea europaea L.) under cover crops management systems in the tropical region of Brazil. Revista Brasileira de Ciência do Solo, v. 44, 2020.

BLAKE, G. R.; HARTGE, K. H. Bulk density. In: KLUTE, A. (Ed.). Methods of soil analysis: part 1: physical and mineralogical methods. 2nd ed. Madison: Soil Science Society of America, 1986. p. 363-375. 
CARDOSO, E. L.; SILVA, M. L. N.; CURI, N.; FERREIRA, M. M.; FREITAS, D. A. F. Qualidade química e física do solo sob vegetação arbórea nativa e pastagens no pantanal sul-mato-grossense. Revista Brasileira de Ciência do Solo, v. 35, n. 2, p. 613-622, 2011.

CARNEIRO, M. A. C.; SOUZA, E. D.; REIS, E. F.; PEREIRA, H. S.; AZEVEDO, W. R. Atributos físicos, químicos e biológicos de solo de Cerrado sob diferentes sistemas de uso e manejo. Revista Brasileira de Ciência do Solo, v. 33, n. 1, p. 147-157, 2009.

COSTA, M. A. T.; TORMENA, C. A.; LUGÃO, S. M. B.; FIDALSKI, J.; NASCIMENTO, W. G.; MEDEIROS, F. M. Resistência do solo à penetração e produção de raízes e de forragem em diferentes níveis de intensificação do pastejo. Revista Brasileira de Ciência do Solo, v. 36, n. 3, p. 993-1004, 2012.

CUNHA, N. R. S.; LIMA, J. E.; GOMES, M. F. M.; BRAGA, M. J. A. Intensidade da exploração agropecuária como indicador da degradação ambiental na região dos cerrados, Brasil. Revista de Economia e Sociologia Rural, v. 46, n. 2, p. 291-323, 2008.

FERREIRA, D. F. Sisvar: A computer statistic alanalysis system. Ciência e Agrotecnologia, v. 35, n. 6, p. 1039-1042, 2011.

FREITAS, P. L.; POLIDORO, J. C.; SANTOS, H. G.; PRADO, R. B.; CALDERANO, S. B.; GREGORIS, G.; MANZATTO, C. V.; DOWICH, I.; BERNARDI, A. C. C. Identificação e caracterização físico-química de Latossolos de textura arenosa e média da região oeste da Bahia. Cadernos de Geociências, v. 11, n. 1, p. 82-92, 2014.

HICKMANN, C.; COSTA, L. M.; SCHAEFER, C. E. G. R.; FERNANDES, R. B. A.; ANDRADE, C. L. T. Atributos físico-hídricos e carbono orgânico de um Argissolo após 23 anos de diferentes manejos. Revista Caatinga, v. 25, n. 1, p. 128-136, 2012.

JORGE, R. F.; ALMEIDA, C. X.; BORGES, E. N.; PASSOS, R. R. Distribuição de poros e densidade em Latossolos submetidos a diversos sistemas de uso e manejo. Bioscience Journal, v. 28, supplement. 1, p. 159-169, 2012.

KER, J. C. Latossolos do Brasil: uma revisão. Geonomos, v. 5, n. 1, p.17-40, 1997.

KLUTE, A. (Ed.). Methods of soil analysis: physical and mineralogical methods. 2.ed. Madison: American Society of Agronomy, 1986. 1188p.

LEROY, B. L. M., HERATH, H. M. S. K., SLEUTEL, S., DE NEVE, S., GABRIELS, D., REHEUL, D., MOENS, M. The quality of exogenous organic matter: short-term effects on soil physical properties and soil organic matter fractions. Soil Use and Management, v. 24, n. 2, p. 139-147, 2008.

MANZATTO, C. V.; FREITAS JUNIOR, E.; PERES, J. R. R. Uso agrícola dos solos brasileiros. (ed.). - Rio de Janeiro: Embrapa Solos, 2002. 174p.

MARCHÃO, R. L.; BALBINO, L. C.; SILVA, E. M.; SANTOS JUNIOR, J. D. G.; SÁ, M. A. C.; VILELA, L. BECQUER, T. Qualidade física de um Latossolo Vermelho sob sistemas de integração lavoura-pecuária no Cerrado. Pesquisa Agropecuária Brasileira, v. 42, n. 6, p. 873-882, 2007.

MOREIRA, W. H.; BETIOLI JUNIOR, E.; PETEAN, L. P.; TORMENA, C. A.; ALVES, S. J.; COSTA, M. A. T.; FRANCO, H. H. S. Atributos físicos de um Latossolo Vermelho distroférrico em sistema de integração lavourapecuária. Revista Brasileira de Ciências do Solo, v. 36, n. 2, p. 389-400, 2012.

NAIR, P. K. R. Agroecosystem management in the 21st century: It is time for a paradigm shift. Journal of Tropical Agriculture, v. 46, n. 1, p. 1-12, 2008.

OLIVEIRA, D. M. S.; LIMA, R. P.; JAN VERBURG, E. E. Qualidade física do solo sob diferentes sistemas de manejo e aplicação de dejeto líquido suíno. Revista Brasileira de Engenharia Agrícola e Ambiental, v. 19, n. 3, p. 280-285, 2015. 
PEZARICO, C. R.; VITORINO, A. C. T.; MERCANTE, F. M.; DANIEL, O. Indicadores de qualidade do solo em sistemas agroflorestais. Revista de Ciências Agrárias, v. 56, n. 1, p. 40-47, 2013.

RAMOS, F. T.; MONARI, Y. C.; NUNES, M. C. M.; CAMPOS, D. T. S. C.; RAMOS, D. T. Indicadores de qualidade em um Latossolo Vermelho-Amarelo sob pastagem extensiva no pantanal matogrossense. Revista Caatinga, v. 23, n. 1, p. 112-120, 2010.

RAMOS, M. R.; FAVARETTO, N.; DIECKOW, J.; DEDECK, R. A.; VEZZANI, F. M.; ALMEIDA, L.; SPERRIN, M. Soil, water and nutrient loss under conventional and organic vegetable production managed in small farms versus forest system. Journal of Agriculture and Rural Development in the Tropics and Subtropics, v. 115, p.131-140, 2014.

REYNOLDS, W. D.; ELRICK, D. E. A laboratory and numerical assessment of the Guelph permeameter method. Soil Science, v. 144, n. 4, p. 282-299, 1987.

ROSSETTI, K. V.; CENTURION, J. F. Sistemas de manejo e atributos físico-hídricos de um Latossolo Vermelho cultivado com milho. Revista Brasileira de Engenharia Agrícola e Ambiental, v. 17, n. 5, p. 472 479, 2013.

SANTOS, R. D.; LEMOS, R. C.; SANTOS, H. G.; KER, J. C.; ANJOS, L. H. C. Manual de descrição e coleta de solo no campo. 6.ed. rev. e ampl. Viçosa-MG: Sociedade Brasileira de Ciência do Solo: UFV; [Rio de Janeiro]: Embrapa Solos: UFRRJ, 2013. 102p.

SOLINO, A. J. S.; FERREIRA, R. O.; FERREIRA, R. L. F.; NETO, S. E. A.; NEGREIROS, J. R. S. Cultivo orgânico de rúcula em plantio direto sob diferentes tipos de coberturas e doses de composto. Revista Caatinga, v. 23, n. 2, p. 18-24, 2010.

SOUSA, D. M. G.; LOBATO, E. Cerrado: correção do solo e adubação. Planaltina, DF: Embrapa Cerrados, 2004. $416 \mathrm{p}$.

STOLF, R.; MURAKAMI, J. H.; BRUGNARO, C.; SILVA, L. G.; SILVA, L. D. F.; MARGARIDO, L. A. C. Penetrômetro de impacto stolf - programa computacional de dados em Excel-VBA. Revista Brasileira de Ciência do Solo, v. 38, n. 3, p.774-782, 2014.

TORRES, J. L. R.; FABIAN, A. J.; PEREIRA, M. G. Alterações dos atributos físicos de um Latossolo Vermelho submetido a diferentes sistemas de manejo. Ciência e Agrotecnologia, v. 35, n. 3, p. 437-445, 2011.

TORRES, J. L. R.; PEREIRA, M. G.; ASSIS, R. L.; SOUZA, Z. M. Atributos físicos de um Latossolo Vermelho cultivado com plantas de cobertura, em semeadura direta. Revista Brasileira de Ciência do Solo, v. 39, n. 2. P. 428-437, 2015.

UHLAND, R. E. Rapid method for determining soil moisture. Soil Science Society of America Journal, v. 15, p. 391-393, 1951.

VOMOCIL, J. A. Porosity. In: BLACK, C. A.; EVANS, D. D.; ESNMINGER, L. E.; CLARK, F. E. (Ed.). Methods of soil analysis: part 1: physical and mineralogical properties. Madison: American Society of Agronomy, 1965. 299-314p. 